\title{
UNARY SEMIGROUPS WITH AN ASSOCIATE SUBGROUP ${ }^{1}$
}

\author{
Paula M. Martins \\ Universidade do Minho, Braga, Portugal \\ Mario Petrich \\ 21420 Bol, Brač, Croatia
}

1. Introduction and summary. In the semigroup literature, regular semigroups have attracted more attention than other classes of semigroups. But within the class of regular semigroups, there is a wide spectrum of special classes, again some of them more popular than others. We deal in this paper with a class of regular semigroups which has been considered by a limited number of mathematicians. We set out here to show that their structural properties deserve a thorough treatment.

Let $S$ be a semigroup. For $s, t \in S, t$ is an associate of $s$ if $s=s t s$; denote by $A(s)$ the set of all associates of $s$. Let $E(S)$ be the set of all idempotents of $S$ and $C(S)$ the core of $S$, that is the subsemigroup of $S$ generated by $E(S)$. For $z \in E(S)$, Green's $\mathcal{H}$-class $H_{z}$ is an associate subgroup of $S$ if for every $s \in S$, the set $A(s) \cap H_{z}$ is a singleton, say $\left\{s^{*}\right\}$; the mapping $s \rightarrow s^{*}$ is a unary operation on $S$. Even though $z$ is not unique, we call it the zenith of $S$. Also, $z$ is called a medial idempotent if for all $s \in C(S)$, we have $s=s z s$, in which case we call $S$ medial. Under these hypotheses, a structure theorem for such semigroups was established in [4] in terms of an idempotent generated semigroup, a group and a single homomorphism.

The parameters of the relevant construction consist of an idempotent generated regular semigroup $C$ with a medial idempotent $w$, a group $G$ and a homomorphism of $G$ into the group of automorphisms of $w C w$. The semigroup $C$ is isomorphic to the core $C(S)$ while the group $G$ is isomorphic to an associate subgroup. Hence the core of $S$ and a single maximal subsemigroup, the associate subgroup, and the action of the latter on a part of the former determine the structure of the whole semigroup, a remarkable phenomenon. In some special cases, this construction reduces to the semidirect product.

2. Terminology and notation. For any semigroup $S$, we use the following notation: in addition to $E(S)$ and $C(S)$ defined above,

$G(S)$ - the set of completely regular elements of $S$ (the union of all subgroups of $S$ ),

\footnotetext{
${ }^{1}$ This work was partly supported by F.C.T. through the Centro de Matemática da Universidade do Minho, Braga, Portugal
} 
$\mathcal{A}(S)$ - the group of automorphisms of $S$,

$V(s)=\{t \in S \mid s=s t s, t=t s t\}$ for any $s \in S$.

If $\rho$ is an equivalence relation on $S$ and $s \in S, \rho s$ denotes the $\rho$-class of $s$.

3. Axioms (A1) - (A7). A semigroup $S$ with a unary operation $s \rightarrow s^{*}$ will be referred to as a $*$-semigroup.

For $*$-semigroups, we will consider the following axioms. For all $s, t \in S$,
(A1) $s=s s^{*} s$,
(A5) $s^{*} t^{*}=\left(s^{*} t^{*}\right)^{* *}$,
(A2) $s^{*} s^{* *}=t^{* *} t^{*}$
(A6) $s=s t^{*} s \Rightarrow s^{*}=t^{*}$
(A3) $(s t)^{*}=t^{*} s^{*}$
(A7) $s^{*}=s^{* * *}$.
(A4) $(s t)^{* *}=s^{* *} t^{* *}$,

The common value in (A2) will be called the zenith of $S$ and denoted by $z$.

We start with a link between semigroups with an associate subgroup and *-semigroups.

Theorem 3.1. Let $S$ be a semigroup, $z \in E(S)$, and assume that $H_{z}$ is an associate subgroup of $S$. For every $s \in S$ define $s^{*}$ by

$$
A(s) \cap H_{z}=\left\{s^{*}\right\} .
$$

Then $S$ is a *-semigroup satisfying axioms (A1), (A5) and (A6).

Conversely, let $S$ be a *-semigroup satisfying axioms (A1), (A5) and (A6). Then $S$ has $H_{z}$ as an associate subgroup where $z=s^{*} s^{* *}$ for any $s \in S$ and (1) holds.

Proof. Let $s, t \in S$.

Direct part. Then $s=s s^{*} s$ and (A1) holds. Next $s^{*} t^{*}\left(s^{*} t^{*}\right)^{*} \in E(S) \cap$ $H_{z} \overline{=\{z\} \text { so that }}$

$$
\left(s^{*} t^{*}\right)^{*}\left(s^{*} t^{*}\right)\left(s^{*} t^{*}\right)^{*}=\left(s^{*} t^{*}\right)^{*} z=\left(s^{*} t^{*}\right)^{*}
$$

and hence $s^{*} t^{*} \in A\left(\left(s^{*} t^{*}\right)^{*}\right) \cap H_{z}=\left\{\left(s^{*} t^{*}\right)^{* *}\right\}$ and (A5) is satisfied. Finally, if $s=s t^{*} s$, then $t^{*} \in A(s) \cap H_{z}=\left\{s^{*}\right\}$ and (A6) holds.

Converse. Let $S^{*}=\left\{s^{*} \mid s \in S\right\}$. By (A1) and (A6), we have that $A(s) \cap$ $S^{*}=\left\{s^{*}\right\}$ and by (A5) that $S^{*}$ is a subsemigroup of $S$. By ([1], Theorem 2), $S^{*}$ is a maximal subgroup of $S$. Axiom (A1) implies that $s^{*}=s^{*} s^{* *} s^{*}$ so that $s^{*} s^{* *}, s^{* *} s^{*} \in E(S)$. But $s^{*} s^{* *}, s^{* *} s^{*} \in S^{*}$. Letting $S^{*}=H_{z}$, we have $s^{*} s^{* *}=z=s^{* *} s^{*}$.

Lemma 3.2. (A1), (A2) $\Rightarrow(\mathrm{A} 7)$. 
Proof. In fact,

$$
\begin{aligned}
s^{*} & =s^{*} s^{* *} s^{*}=s^{*}\left(s^{* *} s^{* * *} s^{* *}\right) s^{*}=\left(s^{*} s^{* *}\right) s^{* * *}\left(s^{* *} s^{*}\right) \\
& =\left(s^{* * *} s^{* * *}\right) s^{* * *}\left(s^{* * * *} s^{* * *}\right)=s^{* * *} .
\end{aligned}
$$

Lemma 3.3. (A1) - (A3) $\Rightarrow(\mathrm{A} 4)-(\mathrm{A} 6)$.

Proof. Indeed,

$$
(s t)^{* *}=\left(t^{*} s^{*}\right)^{*}=s^{* *} t^{* *}
$$

and thus (A4) holds. By (A4) and Lemma 3.2, we have

$$
\left(s^{*} t^{*}\right)^{* *}=s^{* * *} t^{* * *}=s^{*} t^{*}
$$

and (A5) is satisfied. If $s=s t^{*} s$, then

$$
\begin{aligned}
s^{*} & =s^{*} s^{* *} s^{*} & & \text { by }(\mathrm{A} 1) \\
& =s^{*} s^{* *} t^{* * *} s^{* *} s^{*} & & \text { by }(\mathrm{A} 4) \\
& =t^{*} t^{* *} t^{* * *} t^{* *} t^{*} & & \text { by }(\mathrm{A} 2) \\
& =t^{*} & & \text { by }(\mathrm{A} 1)
\end{aligned}
$$

which gives (A6) .

Lemma 3.4. (A1), (A2), (A4) $\Rightarrow(\mathrm{A} 3)$.

Proof. Indeed,

$$
\begin{aligned}
(s t)^{* *}\left(t^{*} s^{*}\right)^{* *}(s t)^{* *} & =s^{* *} t^{* *} t^{* * *} s^{* * *} s^{* *} t^{* *} & & \text { by }(\mathrm{A} 4) \\
& =s^{* *} s^{* * *} s^{* *} t^{* *} t^{* * *} t^{* *} & & \text { by }(\mathrm{A} 2) \text { applied to } s^{*} \text { and } t^{*} \\
& =s^{* *} t^{* *} & & \text { by }(\mathrm{A} 1) \\
& =(s t)^{* *} & & \text { by }(\mathrm{A} 4)
\end{aligned}
$$

and hence

$$
\begin{aligned}
(s t)^{*} & =(s t)^{*}(s t)^{* *}(s t)^{*} & & \text { by }(\mathrm{A} 1) \\
& =(s t)^{*}(s t)^{* *}\left(t^{*} s^{*}\right)^{* *}(s t)^{* *}(s t)^{*} & & \text { by the above } \\
& =\left(t^{*} s^{*}\right)^{* *}\left(t^{*} s^{*}\right)^{* * *}\left(t^{*} s^{*}\right)^{* *}\left(t^{*} s^{*}\right)^{* * *}\left(t^{*} s^{*}\right)^{* *} & & \text { by }(\mathrm{A} 2) \\
& =\left(t^{*} s^{*}\right)^{* *} & & \text { by (A1) } \\
& =t^{* * *} s^{* * *} & & \text { by (A4) } \\
& =t^{*} s^{*} & & \text { by Lemma 3.2. }
\end{aligned}
$$

Lemma 3.5. (A1), (A5), (A6) $\Rightarrow(\mathrm{A} 2)$.

Proof. By the proof of Theorem 3.1, $S^{*}=H_{z}$ with $z=s^{*} s^{* *}=s^{* *} s^{*}$ for any $s \in S$. Then for every $s, t \in S$, we have $s^{*} s^{* *}=z=t^{* *} t^{*}$ and (A2) holds. 
4. Properties of *-semigroups. In this section, $S$ stands for $a *-$ semigroup.

Notation. For any $s \in S$, let

$$
s^{+}=s(s s)^{*} s, \quad s^{\circ}=\left(s^{+}\right)^{2} .
$$

The idempotents of $S$ can now be easily characterized.

Lemma 4.1. Let $S$ satisfy (A1). Then $E(S)=\left\{s^{\circ} \mid s \in S\right\}$.

Proof. If $e \in E(S)$, then $e^{\circ}=\left[e(e e)^{*} e\right]^{2}=e^{2}=e$. Conversely, for any $s \in S$, we have

$$
\begin{aligned}
s^{\circ} s^{\circ} & =s(s s)^{*}\left[s s(s s)^{*} s s\right](s s)^{*} s s(s s)^{*} s \\
& =s(s s)^{*}\left[s s(s s)^{*} s s\right](s s)^{*} s=\left[s(s s)^{*} s\right]^{2}=s^{\circ} .
\end{aligned}
$$

Lemma 4.2. Let $S$ satisfy axiom (A1) and let $s, t \in S$.

(i) $s \mathcal{L} t \Longleftrightarrow s=s t^{*} t, t=t s^{*} s \Longleftrightarrow s^{*} s \mathcal{L} t^{*} t$.

(ii) $s \mathcal{R} t \Longleftrightarrow s=t t^{*} s, t=s s^{*} t \Longleftrightarrow s s^{*} \mathcal{R} t t^{*}$.

(iii) $s \mathcal{H} t \Longleftrightarrow s=t t^{*} s t^{*} t, t=s s^{*} t s^{*} s$.

(iv) $s \mathcal{H} t, s^{*}=t^{*} \Longrightarrow s=t$.

Proof. (i) For $s, t \in S$, we have

$s \mathcal{L} t \Leftrightarrow$ there exist $u, v \in S^{1}$ such that $s=u t, \quad t=v s$

$\Leftrightarrow s=s t^{*} t, \quad t=t s^{*} s$

$\Leftrightarrow \quad s^{*} s=s^{*} s t^{*} t, \quad t^{*} t=t^{*} t s^{*} s$

$\Leftrightarrow \quad$ there exist $w, x \in S^{1}$ such that $s^{*} s=w t^{*} t, \quad t^{*} t=x s^{*} s$

$\Leftrightarrow s^{*} s \mathcal{L} t^{*} t$

(ii) This is the dual of part (i).

(iii) This follows easily from parts (i) and (ii).

(iv) If $s \mathcal{H} t$ and $s^{*}=t^{*}$, then by parts (i) and (ii), we get $s=t t^{*} s=$ $t s^{*} s=t$.

Completely regular elements of a *-semigroup satisfying only axiom (A1) admit multiple characterizations as follows.

Theorem 4.3. Let $S$ satisfy (A1). The following conditions on $s \in S$ are equivalent. 
(i) $s$ is completely regular.

(ii) $s=s^{+} s=s s^{+}$.

(iii) $s=s^{\circ} s=s s^{\circ}$.

(iv) $s=s s(s s)^{*} s(s s)^{*} s s$.

(v) $s \mathcal{H} s^{\circ}$.

Moreover, if these conditions are satisfied, then

$$
s^{\circ}=s^{+}, \quad s^{-1}=s(s s)^{*} s(s s)^{*} s .
$$

Proof. (i) $\Rightarrow$ (ii) Then $s \mathcal{L} s^{2}$ which by Lemma 4.2(i) yields that

$$
s=s\left(s^{2}\right)^{*} s^{2}=s^{+} s
$$

and dually $s=s s^{+}$.

(ii) $\Rightarrow$ (iii) Indeed,

$$
s=s^{+} s=s^{+} s^{+} s=s^{\circ} s
$$

and dually $s=s s^{\circ}$.

(iii) $\Rightarrow$ (iv) In fact,

$$
\begin{aligned}
s & =s s^{*} s=\left(s s^{\circ}\right) s^{*}\left(s^{\circ} s\right) \\
& =s\left[s(s s)^{*} s\right]\left[s(s s)^{*} s\right] s^{*}\left[s(s s)^{*} s\right]\left[s(s s)^{*} s\right] s \\
& =s s(s s)^{*} s(s s)^{*} s s .
\end{aligned}
$$

(iv) $\Rightarrow$ (i) Then $s \in s^{2} S s^{2}$ and is by ([6], Proposition II.1.3) completely regular.

(iii) $\Rightarrow(\mathrm{v})$ The hypothesis implies that $s \in s^{\circ} S \cap S s^{\circ}$ and the definition of $s^{\circ}$ yields that $s^{\circ} \in s S \cap S s$. Hence $s \mathcal{H} s^{\circ}$.

$(\mathrm{v}) \Rightarrow$ (i) This is well known, see [6].

Now assume that the above conditions are fulfilled. Then

$$
s^{+}=s(s s)^{*} s=s(s s)^{*} s s^{+}=s^{+} s^{+}=s^{\circ} .
$$

Next let $\bar{s}=s(s s)^{*} s(s s)^{*} s$. We obtain

$$
\bar{s} s s=\left[s(s s)^{*} s(s s)^{*} s\right] s s=\left(s(s s)^{*}\right) s^{+} s s=\left(s(s s)^{*}\right) s s=s^{+} s=s
$$

and $s \in \bar{s} S$ and dually $s \in S \bar{s}$. By definition $\bar{s} \in s S \cap S s$ and hence $\bar{s} \mathcal{H} s$. Now $s \mathcal{H} s^{2}$ by Lemma 4.2(iii) implies that

$$
s=\left(s s(s s)^{*}\right) s\left((s s)^{*} s s\right)=s \bar{s} s
$$

and thus $\bar{s}=s^{-1}$. 
The next proposition relates the unary operation on $S$ with the least group congruence on $S$ in a generic way.

Proposition 4.4. Let $S$ be a *-semigroup satisfying axioms (A1), (A5), (A6). Define a relation $\theta$ by

$$
s \theta t \Longleftrightarrow s^{*}=t^{*} \quad(s, t \in S) .
$$

Then the congruence $\theta^{\sharp}$ generated by $\theta$ is the least group congruence $\sigma$ on $S$ and $S / \sigma \cong H_{z} /\left(\left.\sigma\right|_{H_{z}}\right)$.

Proof. Since $S$ is a regular semigroup, so is $S / \theta^{\sharp}$. Let $e \in E(S)$. By Lemmas 3.2 and 3.5 , we get that $e \theta e^{* *}$ and thus $e \theta^{\sharp} e^{* *}$ whence $e e^{*} \theta^{\sharp} e^{* *} e^{*}=z$ and similarly $e^{*} e \theta^{\sharp} z$. Hence, by Theorem 3.1,

$$
e=e e^{*} e \theta^{\sharp} e^{*} e e=e^{*} e \theta e^{*} e^{* *}=z .
$$

Hence all idempotents of $S$ are $\theta^{\sharp}$-related to $z$. In view of Lallement's lemma, we conclude that $S / \theta^{\sharp}$ is a group, that is $\theta^{\sharp}$ is a group congruence.

Next let $\rho$ be a group congruence on $S$ and let $s \theta t$. Then $s^{*}=t^{*}$ and $s=s s^{*} s$ and $t=t t^{*} t$ imply

$$
\rho s=\left(\rho s^{*}\right)^{-1}=\left(\rho t^{*}\right)^{-1}=\rho t
$$

and thus $\theta \subseteq \rho$. But then also $\theta^{\sharp} \subseteq \rho$ proving the minimality of $\theta^{\sharp}$.

By Lemmas 3.5 and 3.2, we have $s^{*}=s^{* * *}$ for any $s \in S$ and that $s \theta s^{* *}$ and finally $s \theta^{\sharp} s^{* *}$, which implies the last assertion of the proposition.

Proposition 4.5. Let $S$ satisfy axioms (A1) - (A3) .

(i) $E(S)=\left\{s^{+} \mid s s^{*} \in V\left(s^{*} s\right)\right\}$.

(ii) $C(S)=\left\{s \in S \mid s^{*}=z\right\}=\left\{s^{+} \mid s \in S\right\}=\{e f \mid e, f \in E(S)\}$.

(iii) $G(S)=\left\{s \in S \mid s s^{*} \in V\left(s^{*} s\right)\right\}$.

(iv) $C(S) \cap G(S)=E(S)$.

Proof. (i) If $e \in E(S)$, then $e=e e^{*} e=e(e e)^{*} e=e^{+}$and

$$
\left(e e^{*}\right)\left(e^{*} e\right)\left(e e^{*}\right)=e(e e)^{*} e e^{*}=e e^{*} e e^{*}=e e^{*}
$$

and similarly $e^{*} e=\left(e^{*} e\right)\left(e e^{*}\right)\left(e^{*} e\right)$ so that $e e^{*} \in V\left(e^{*} e\right)$.

Conversely, let $s s^{*} \in V\left(s^{*} s\right)$. Then

$$
s s^{*}=\left(s s^{*}\right)\left(s^{*} s\right)\left(s s^{*}\right)
$$

whence

$$
s^{+} s^{+}=s(s s)^{*} s s(s s)^{*} s=\left(s s^{*}\right)\left(s^{*} s\right)\left(s s^{*}\right)\left(s^{*} s\right)=s^{+} .
$$


(ii) For every $e \in E(S)$, we have

$$
e^{*}=e^{*} e^{* *} e^{*}=e^{*}(e e)^{* *} e^{*}=e^{*} e^{* *} e^{* *} e^{*}=z z=z .
$$

For $p \in C(S)$, we have $p=e_{1} e_{2} \cdots e_{n}\left(e_{i} \in E(S)\right)$ and by (A3),

$$
p^{*}=e_{n}^{*} \cdots e_{2}^{*} e_{1}^{*}=z
$$

and $C(S) \subseteq\left\{s \in S \mid s^{*}=z\right\}$.

Let $s \in S$ and $s^{*}=z$. Then

$$
s=s s^{*} s=s z s=(s z)(z s)=\left(s s^{*}\right)\left(s^{*} s\right)=s^{+}
$$

and $\left\{s \in S \mid s^{*}=z\right\} \subseteq\left\{s^{+} \mid s \in S\right\}$. For any $s \in S$, we have $s s^{*}, s^{*} s \in E(S)$ and thus $\left\{s^{+} \mid s \in S\right\} \subseteq\{e f \mid e, f \in E(S)\}$. Finally by definition, we have $\{e f \mid e, f \in E(S)\} \subseteq C(S)$.

(iii) If $s \in G(S)$, then by Theorem 4.3, we have $s=s^{+} s=s s^{+}$which evidently implies (1) and its counterpart for $s^{*} s$ so that $s s^{*} \in V\left(s^{*} s\right)$. The converse follows essentially the same way.

(iv) This is an obvious consequence of parts (i) - (iii).

Since

$$
\begin{aligned}
\left(s^{+}\right)^{3} & =\left[s(s s)^{*} s\right]\left[s(s s)^{*} s\right]\left[s(s s)^{*} s\right] \\
& =s(s s)^{*}\left[s s(s s)^{*} s s\right](s s)^{*} s=s^{+} s^{+}
\end{aligned}
$$

we see that $\left(s^{+}\right)^{2}=\left(s^{+}\right)^{3}$ which in view of Proposition 4.5(ii) shows that $C(S)$ is periodic. By Proposition 4.5(iv) we have that $C(S)$ is combinatorial. Both of these statements were proved in ([2], Theorem 1.1).

Lemma 4.6. Let $S$ be a *-semigroup satisfying axioms (A1) - (A3). For $s, t \in S$, we have

$$
s \leq t \Longleftrightarrow s=s s^{*} t=t s^{*} s .
$$

Proof. By Proposition 4.5(ii), for $e \in E(S)$, we have $e^{*}=z$, and thus

$$
\begin{aligned}
s \leq t & \Rightarrow s=e t=t f \text { for some } e, f \in E(S) \\
& \Rightarrow\left\{\begin{array}{l}
s=e t t^{*} t=e t t^{*} e^{*} t=e t(e t)^{*} t=s s^{*} t \\
s=t t^{*} t f=t f^{*} t^{*} t f=t(t f)^{*} t f=t s^{*} s
\end{array}\right. \\
& \Rightarrow s \leq t .
\end{aligned}
$$

5. Characterizations. It was proved in [4] that a regular semigroup $S$ with an associate subgroup $G$ whose identity is medial can be constructed by 
an action of $G$ on a part of the core $C(S)$ of $S$. We state this construction below and establish a number of its properties. This serves primarily for understanding of the structure of these semigroups, but it will be also prove useful for general manipulation.

We start with a multiple characterization of the basic conditions in this section.

Lemma 5.1. Let $S$ be a $*$-semigroup satisfying axioms (A1), (A5), (A6) and write $z$ for the zenith of $S$. Then the following statements are equivalent.

(i) $z$ is medial.

(ii) $S$ satisfies axiom (A3).

(iii) $p^{*}=z$ for all $p \in C(S)$.

(iv) The relation $\theta$ in Proposition 4.4 is a congruence.

Proof. (i) $\Rightarrow$ (ii) For $s, t \in S$, we have

$$
\begin{aligned}
(s t)\left(t^{*} s^{*}\right)(s t) & =s s^{*} s t t^{*} t^{* *} t^{*} s^{*} s t t^{*} t & & \text { by }(\mathrm{A} 1) \\
& =s\left(s^{*} s t t^{*}\right) z\left(s^{*} s t t^{*}\right) t & & \text { by Theorem } 3.1 \\
& =s s^{*} s t t^{*} t & & \text { by mediality of } z \\
& =s t & & \text { by (A1) }
\end{aligned}
$$

and thus $t^{*} s^{*} \in A(s t)$ Then by (A5) and (A6), we have $t^{*} s^{*} \in A(s t) \cap S^{*}=$ $\left\{(s t)^{*}\right\}$.

(ii) $\Rightarrow$ (iii) This follows from Lemma 3.5 and Proposition 4.5(ii).

(iii) $\Rightarrow$ (i) If $p \in C(S)$, then $p=p p^{*} p=p z p$.

(ii) $\Rightarrow$ (iv) This is obvious.

(iv) $\Rightarrow$ (ii) For any $s \in S$, by Lemmas 3.2 and 3.5, we get $s \theta s^{* *}$. Hence $(s t)^{*}=\left(s^{* *} t\right)^{*}$ for any $s, t \in S$. By Lemma 3.5, we have that

$$
s^{*} t\left(t^{*} s^{* *}\right) s^{*} t=s^{*} t t^{*}\left(s^{* *} s^{*}\right) t=s^{*} t t^{*} t=s^{*} t
$$

which by (A6) yields that $\left(s^{*} t\right)^{*}=t^{*} s^{* *}$. Using this formula, we get $\left(\left(s^{*}\right)^{*} t\right)^{*}=$ $t^{*} s^{* * *}=t^{*} s^{*}$ and thus $(s t)^{*}=t^{*} s^{*}$.

The equivalence of parts (i) and (ii) in Lemma 5.1 was proved by a longer argument in ([1], Theorem 4).

The following construction represents the basic device for a description of the semigroups discussed throughout this section.

We quote from [4]. 
Construction. Let $C$ be an idempotent generated semigroup with a medial idempotent $w$. Let $G$ be a group and $\zeta: G \rightarrow \mathcal{A}(w C w)$ be a homomorphism and write $\zeta: g \rightarrow \zeta_{g}$. On the set

$$
\left\{(x, g, a) \in C w \times G \times w C \mid \zeta_{g}(a w)=w x\right\}
$$

define a multiplication by

$$
(x, g, a)(y, h, b)=\left(x \zeta_{g}(a y), g h, \zeta_{h^{-1}}(a y) b\right)
$$

and denote the resulting structure by $[C, G ; w, \zeta]$.

Additionally, we introduce a unary operation

$$
(x, g, a)^{*}=\left(w, g^{-1}, w\right) .
$$

Toward the proof of the characterization theorem (Theorem 5.7 below), we first establish lemmas each of which implies one of the implications in the proof of the theorem. These lemmas state explicitly the transition of one characterization to another thereby contributing to a better understanding of the content of various parts of the theorem.

Lemma 5.2. Let $S$ be a semigroup with an associate subgroup $H_{z}$ whose identity $z$ is medial. Define

$$
\zeta: g \longrightarrow \zeta_{g} \quad\left(g \in H_{z}\right)
$$

where

$$
\zeta_{g}: p \longrightarrow g p g^{-1} \quad(p \in z C(S) z) .
$$

Then $\left[C(S), H_{z} ; z, \zeta\right]$ is defined. For every $s \in S$, define $s^{*}$ by $A(s) \cap H_{z}=$ $\left\{s^{*}\right\}$. Then the mapping

$$
\varphi: s \longrightarrow\left(s s^{*}, s^{* *}, s^{*} s\right) \quad(s \in S)
$$

is an isomorphism of $S$ onto $\left[C(S), H_{z} ; z, \zeta\right]$.

Proof. This is the content of ([4], Theorem 4).

Lemma 5.3. Let $S=[C, G ; w, \zeta]$. Then $S$ satisfies axioms (A1) - (A3).

Proof. Let $(x, g, a),(y, h, b) \in S$. Then

$$
\begin{aligned}
(x, g, a)(x, g, a)^{*}(x, g, a) & =(x, g, a)\left(w, g^{-1}, w\right)(x, g, a) \\
& =\left(x \zeta_{g}(a w), 1, \zeta_{g}(a w) w\right)(x, g, a) \\
& =(x w x, 1, w x w)(x, g, a)=(x, 1, w x)(x, g, a) \\
& =\left(x w x x, g, \zeta_{g^{-1}}(w x x) a\right)=(x, g, a w a)=(x, g, a)
\end{aligned}
$$


and therefore (A1) is satisfied. Next,

$$
\begin{aligned}
(x, g, a)^{*}(x, g, a)^{* *} & =\left(w, g^{-1}, w\right)(w, g, w)=(w, 1, w), \\
(y, h, b)^{* *}(y, h, b)^{*} & =(w, h, w)\left(w, h^{-1}, w\right)=(w, 1, w)
\end{aligned}
$$

and so (A2) holds. Finally,

$$
\begin{aligned}
(y, h, b)^{*}(x, g, a)^{*} & =\left(w, h^{-1}, w\right)\left(w, g^{-1}, w\right) \\
& =\left(w,(g h)^{-1}, w\right) \\
& =\left(x \zeta_{g}(a y), g h, \zeta_{h^{-1}}(a y) b\right)^{*} \\
& =((x, g, a)(y, h, b))^{*}
\end{aligned}
$$

and (A3) is satisfied as well.

Lemma 5.4. Let $S$ be a *-semigroup satisfying axioms (A1) - (A3). Then the mapping

$$
\chi: s \rightarrow s^{* *} \quad(s \in S)
$$

is a retraction of $S$ onto $H_{z}$, where $z=s^{*} s^{* *}$ for every $s \in S$, such that $s=s(\chi s)^{-1} s$ for every $s \in S$.

Proof. By Lemma 3.3, $\chi$ is a homomorphism. Let $z=s^{*} s^{* *}$ for any $s \in S$. Then by (A1),

$$
z z=s^{*} s^{* *} s^{*} s^{* *}=s^{*} s^{* *}=z,
$$

and so $H_{z}$ is a maximal subgroup of $S$.

For any $g \in H_{z}$, we get

$$
\begin{aligned}
& g^{*} g=g^{*} g z=g^{*} g g^{*} g^{* *}=g^{*} g\left(g^{*} g\right)^{*}=z \\
& g g^{*}=g z g^{*}=g g^{*} g^{* *} g^{*}=g g^{*}\left(g g^{*}\right)^{*}=z \\
& z g^{*}=g^{*} g^{* *} g^{*}=g^{*} \\
& g^{*} z=g^{*} g^{*} g^{* *}=\left(g^{*} g g\right)^{*}=(z g)^{*}=g^{*}
\end{aligned}
$$

thus $g^{*} \in H_{z}$ and $g^{-1}=g^{*}$. For any $g \in H_{z}$, we have

$$
\chi g=g^{* *}=\left(g^{-1}\right)^{-1}=g .
$$

The fact that $z^{*}=z^{-1}=z$ gives that for every $s \in S$,

$$
\begin{aligned}
s^{* *} s^{* * *} & =z, s^{*} s^{* *}=z, \quad z s^{* *}=s^{* *} s^{* * *} s^{* *}=s^{* *}, \\
s^{* *} z & =s^{* *} z^{*}=s^{* *}\left(s^{*} s^{* *}\right)^{*}=s^{* *} s^{* * *} s^{* *}=s^{* *}
\end{aligned}
$$


and so $s^{* *} \in H_{z}$. It follows that $\chi$ is a retraction of $S$ onto $H_{z}$. In addition, for any $s \in S$ we have $s^{* * *}=\chi\left(s^{*}\right)=s^{*}$ since $s^{*} \in S^{*}=H_{z}$ by the proof of Theorem 3.1, and

$$
s=s s^{*} s=s s^{* * *} s=s\left(s^{* *}\right)^{-1} s=s(\chi s)^{-1} s .
$$

Let $\rho$ be a congruence on a semigroup $S$. A subset $T$ of $S$ is a transversal of $\rho$ if $T$ intersects each $\rho$-class exactly once. If also for every element $s$ of $S, T$ contains exactly one associate of $s$, then $T$ is an associate transversal of $\rho$.

Lemma 5.5. Let $S$ be a semigroup, $z \in E(S)$ and $\chi$ be a retraction of $S$ onto $H_{z}$ such that $s=s(\chi s)^{-1} s$ for all $s \in S$. The kernel $\rho=\operatorname{ker} \chi$ is a congruence which has $H_{z}$ as an associate transversal.

Proof. The relation $\rho$ is obviously a congruence on $S$. We will prove that for any $s \in S$,

$$
A(s) \cap H_{z}=\left\{(\chi s)^{-1}\right\}, \quad \rho s \cap H_{z}=\{\chi s\} .
$$

We have $(\chi s)^{-1} \in H_{z} \cap A(s)$. If $\chi t \in A(s)$, then $s=s(\chi t) s$ and thus $\chi s=(\chi s)(\chi t)(\chi s)$ so that $\chi t=(\chi s)^{-1}$. Since $\chi$ is a retraction of $S$ onto $H_{z}$, we have $\chi(\chi s)=\chi s$ and thus $\chi s \in \rho s$. If $t \in H_{z} \cap \rho s$, then $t=\chi t=\chi s$. Hence $H_{z}$ is an associate transversal of $\rho$.

Lemma 5.6. Let $S$ be a semigroup having a congruence $\rho$ for which $H_{z}$ is an associate transversal for some idempotent $z$. Then $z$ is medial.

Proof. For any $s \in S$ define $s^{*}$ by the condition $A(s) \cap H_{z}=\left\{s^{*}\right\}$. By Theorem 3.1 and Lemma 3.5, we get $z=s^{*} s^{* *}=t^{* *} t^{*}$ for all $s, t \in S$, and by Lemma 3.2, we have $s^{*}=s^{* * *}$ for all $s \in S$. For $t^{*} \in \rho s \cap H_{z}$, we have

$$
s=s s^{*} s^{* *} s^{*} s \rho t^{*}\left(s^{*} s^{* *}\right) s^{*} t^{*}=t^{*}\left(t^{* *} t^{*}\right) s^{*} t^{*}=t^{*} s^{*} t^{*}
$$

whence $t^{*} s^{*} t^{*}=t^{*}$. Thus $s^{*}=t^{* *}$ and so $t^{*}=t^{* * *}=s^{* *}$. It follows that $\rho s \cap H_{z}=\left\{s^{* *}\right\}$.

For every $e \in E(S)$, we have $e \rho e^{* *}$ and thus $e=e e \rho e^{* *} e^{* *}$. Since $e^{* *} e^{* *} \in H_{z}$, we have $e^{* *} e^{* *} \in \rho e \cap H_{z}=\left\{e^{* *}\right\}$ and $e^{* *} \in E\left(H_{z}\right)=\{z\}$. For $p \in C(S), p=e_{1} e_{2} \ldots e_{n}\left(e_{i} \in E(S)\right)$, and hence $p^{* *} \rho p \rho e_{1}^{* *} e_{2}^{* *} \ldots e_{n}^{* *}=z$, which implies that $p^{* *}=z$ and thus $p^{*}=p^{*} p^{* *} p^{*}=p^{*} z p^{*}=p^{*} p^{*}$. Hence $p^{*}=z$ and therefore $p=p z p$.

We are finally ready for the desired result.

Theorem 5.7. The following conditions on a semigroup $S$ are equivalent. 
(i) S has an associate subgroup whose identity is medial.

(ii) $S \cong[C, G ; w, \zeta]$ for some parameters $C, G, w$ and $\zeta$.

(iii) $S$ is a *-semigroup satisfying (A1) and any of:

( $\alpha)(\mathrm{A} 2),(\mathrm{A} 3)$;

( $\beta)(\mathrm{A} 2),(\mathrm{A} 4)$;

$(\gamma)$ (A5), (A6) and its zenith $z$ is medial;

$(\delta)$ (A5), (A6) and the relation $\theta$ in Proposition 4.4 is a congruence.

(iv) $S$ has a retraction $\chi$ onto a maximal subgroup of $S$ such that $s=$ $s(\chi s)^{-1} s$ for every $s \in S$.

(v) S has a congruence $\rho$ with an associate transversal which is a maximal subgroup of $S$.

(vi) A maximal subgroup $G$ of $S$ contains an associate of each element of $S$ and is a transversal of the least group congruence on $S$.

Proof. (i) $\Rightarrow$ (ii) This follows from Lemma 5.2.

(ii) $\Rightarrow$ (iii) This follows from Lemmas $3.3-3.5,5.1$ and 5.3.

(iii) $\Rightarrow$ (iv) This was proved in Lemma 5.4.

(iv) $\Rightarrow$ (v) This follows from Lemma 5.5.

(v) $\Rightarrow(\mathrm{vi})$ Let the transversal be $H_{z}$. Since the transversal is a group, $\rho$ is a group congruence. Let $\lambda$ be a group congruence on $S$. For $s \in S$ write

$$
[s]=\rho s \in S / \rho \quad \text { and } \quad \bar{s}=\lambda s \in S / \lambda .
$$

Suppose that $s \rho t$. Then $\left[s^{*}\right]=[s]^{-1}=[t]^{-1}=\left[t^{*}\right]$. Now for $u \in S$ we have $\left[u^{* *}\right]=\left([u]^{-1}\right)^{-1}=[u]$ and so

$$
\left[s^{* *} t^{* * *} s^{* *}\right]=\left[s^{* *}\right]\left[t^{* * *}\right]\left[s^{* *}\right]=[s]\left[t^{*}\right][s]=[s]\left[s^{*}\right][s]=[s]=\left[s^{* *}\right] .
$$

Thus $s^{* *} t^{* * *} s^{* *} \rho s^{* *}$. But since $s^{* *} t^{* * *} s^{* *}, s^{* *} \in H_{z}$ we have $s^{* *} t^{* * *} s^{* *}=s^{* *}$. By Theorem 3.1 we have $t^{* * *}=s^{* * *}$ and it follows, by Lemmas 3.2 and 3.5 , that $t^{*}=s^{*}$. In $S / \lambda$, we have $\bar{s}=\bar{s} \overline{s^{*}} \bar{s}$ and $\bar{t}=\bar{t} \overline{t^{*}} \bar{t}$ which yields $\bar{s}^{-1}=\overline{s^{*}}=\overline{t^{*}}=\bar{t}^{-1}$ so that $\bar{s}=\bar{t}$ and $s \lambda t$. Therefore $\rho \subseteq \lambda$ which proves the minimality of $\rho$.

(vi) $\Rightarrow$ (i) We show first that $G$ is an associate subgroup of $S$. By hypothesis, for every $s \in S$, the set $A(s) \cap G$ is nonempty. Suppose that $u, v \in A(s) \cap G$. Then $s=$ sus $=$ svs which in $\bar{S}=S / \sigma$ becomes $\bar{s}=\bar{s} \bar{u} \bar{s}=$ 
$\bar{s} \bar{v} \bar{s}$ so that $\bar{u}=\bar{v}$. This means that $u \sigma v$. Since $G$ is a transversal of $\sigma$, it follows that $u=v$. Therefore $|A(s) \cap G|=1$ and $G$ is an associate subgroup of $S$. By Lemma 5.6, we conclude that the identity of $G$ is medial.

We denote by $\sigma_{S}$ the least group congruence on any regular semigroup $S$. A monoid $S$ is uniquely unit orthodox if $S$ is orthodox and every element has a unique associate in the group of units of $S$, see [3]. A semigroup $S$ is $F$-regular if $S$ is regular and each class of the least group congruence on $S$ has a greatest element under the natural partial order, see [5].

We now list some supplementary information to that in Theorem 5.7.

Proposition 5.8. Let $S$ be as in Theorem 5.7 having $z$ as zenith.

(i) If the parameters in part (ii) are chosen as in Lemma 5.2, the isomorphism $\varphi$ in Lemma 5.2 is a *-isomorphism.

(ii) In part (iv) for every $s \in S$, we have $\chi s=s^{* *}$.

(iii) The congruence $\rho$ in part (v) is the least group congruence on $S$.

(iv) For every $s \in z S z, s^{*}$ is the unique associate of $s$ in $H_{z}$ and $z S z$ is orthodox. Consequently the semigroup $z S z$ is uniquely unit orthodox.

(v) For every $s \in z S z, s^{* *}$ is the greatest element of the $\sigma_{z S z}$-class of $s$. Consequently the semigroup $z S z$ is F-regular.

Proof. (i) For any $s \in S$, we get by Lemma 5.2,

$$
\varphi s^{*}=\left(s^{*} s^{* *}, s^{* * *}, s^{* *} s^{*}\right)=\left(z,\left(s^{* *}\right)^{-1}, z\right)=(\varphi s)^{*}
$$

and $\varphi$ is a $*$-isomorphism.

(ii) For $s \in S$, we have $(\chi s)^{-1} \in A(s) \cap H_{z}=\left\{s^{*}\right\}$ and $(\chi s) s^{*}=z$. Then $s^{*}(\chi s) s^{*}=s^{*} z=s^{*}$ and thus $\chi s \in A\left(s^{*}\right) \cap H_{z}=\left\{s^{* *}\right\}$ whence the assertion.

(iii) This was established in the proof of the implication (v) $\Rightarrow$ (vi) in Theorem 5.7.

(iv) Since $H_{z}$ is the group of units of $z S z$ it suffices to show that $z S z$ is orthodox. For any $e, f \in E(z S z)$, we have

$$
e f=(e f) z(e f)=(e f)(e f) \text {. }
$$

(v) Let $\sigma=\sigma_{z S z}$ and $s \in z S z$. Then

$$
\begin{aligned}
& s=s s^{*} s=\left(s s^{*}\right) s^{* *}\left(s^{*} s\right) \sigma s^{* *}, \\
& s=z s=s^{* *}\left(s^{*} s\right)=s z=\left(s s^{*}\right) s^{* *}
\end{aligned}
$$


and thus $s \leq s^{* *}$. For any $s, t \in S^{*}$, by Proposition 4.4 and Lemma 5.1, we have

$$
s \sigma_{S} t \Longleftrightarrow s^{*}=t^{*} \Longleftrightarrow s \sigma_{z S z} t
$$

and hence $\left.\sigma_{S}\right|_{z S z}=\sigma$. Next let $t \in z S z$ be such that $t \sigma s$. Theorem 5.7 and Lemma 5.1 imply that $t^{*}=s^{*}$ whence $t^{* *}=s^{* *}$. But then $t \leq t^{* *}$ implies that $t \leq s^{* *}$. Therefore $s^{* *}$ is the greatest element of $\sigma s$. By definition, $z S z$ is $F$-regular.

Corollary 5.9. Let $S=[C, G ; w, \zeta]$. Every subgroup of $S$ can be embedded into $G$. Every group homomorphic image of $S$ is a homomorphic image of $G$.

Proof. This follows from Theorem 5.7(vi), Proposition 5.8(ii) and Lemma 4.2(iv).

So all these properties boil down to properties of a maximal subgroup. It in turn is determined by its identity, that is an idempotent of $S$. Hence we may ask the following question: what are necessary and sufficient conditions on an idempotent $z$ of a (regular) semigroup $S$ in order for $S$ to have $H_{z}$ as an associate subgroup? From Theorem 5.7(vi):

The following conditions on an idempotent $z$ of a semigroup $S$ are equivalent.

(i) $H_{z}$ is an associate subgroup of $S$.

(ii) $H_{z}$ is a transversal of the least group congruence on $S$ and every element of $S$ has an associate in $H_{z}$.

6. Regular semigroups with group transversal. As a generalization of the case considered in the preceding section, we construct here a system of axioms which characterizes the regular semigroups $S$ having a subgroup $G$ which is a transversal of a (necessarily group) congruence on $S$. Relative to the case studied in the preceding section, we drop the requirement that $G$ contains an associate of every element of $S$.

Theorem 6.1. Let $S$ be a regular semigroup.

Let $\rho$ be a congruence on $S$ having a transversal $G$ which is a subgroup of $S$. For every $s \in S$, define $s^{*}$ by $(\rho s)^{-1} \cap G=\left\{s^{*}\right\}$. Then $G=\left\{s^{*} \mid s \in S\right\}$ and the unary operation $s \rightarrow s^{*}$ satisfies the axioms:

$$
\left(\mathrm{A} 1^{*}\right) s^{*}=s^{*} s^{* *} s^{*}
$$

and $(\mathrm{A} 2),(\mathrm{A} 3)$. 
Conversely, let $S$ have a unary operation $s \rightarrow s^{*}$ satisfying these axioms. Define a relation $\rho$ by

$$
s \rho t \Longleftrightarrow s^{*}=t^{*} \quad(s, t \in S) .
$$

Then $\rho$ is a congruence on $S$ and $S^{*}=\left\{s^{*} \mid s \in S\right\}$ is a subgroup of $S$ and a transversal of $\rho$.

Proof. Since the transversal $G$ is a group, $\rho$ is a group congruence with $S / \rho \cong G$ and $G \subseteq H_{z}$ for some $z \in E(S)$. Then $S^{*}=\left\{s^{*} \mid s \in S\right\} \subseteq G$. Let $s \in G$. Then $\rho s^{*}=(\rho s)^{-1}$ so that $\left(\rho s^{*}\right)^{-1}=\rho s$ and

$$
s \in\left(\rho s^{*}\right)^{-1} \cap G=\left\{s^{* *}\right\} .
$$

Hence $s=s^{* *}$. Therefore $G \subseteq S^{*}$ and thus $G=S^{*}$.

Let $s, t \in S$. The definition implies that $\left(\rho s^{*}\right)^{-1}=\rho s^{* *}$ and thus $s^{*} s^{* *} s^{*} \rho s^{*}$. Since $s^{*}, s^{* *} \in G$, we deduce that $\left(\mathrm{A} 1^{*}\right)$ holds. Also $s^{*} s^{* *}$ is contained in the unit $\rho$-class as is $t^{* *} t^{*}$. Since $s^{*} s^{* *}, t^{* *} t^{*} \in G$, it follows that (A2) is satisfied. Finally $\rho t^{*}=(\rho t)^{-1}, \rho s^{*}=(\rho s)^{-1}$ and

$$
\rho\left(t^{*} s^{*}\right)=\left(\rho t^{*}\right)\left(\rho s^{*}\right)=(\rho t)^{-1}(\rho s)^{-1}=(\rho(s t))^{-1}=\rho(s t)^{*}
$$

so that $t^{*} s^{*} \rho(s t)^{*}$ where $t^{*} s^{*},(s t)^{*} \in G$ and thus (A3) holds as well.

Conversely, by (A3) the map: $S \rightarrow S, s \mapsto s^{*}$ is an antihomomorphism, so its kernel $\rho$ is a congruence and its image $S^{*}$ is a subsemigroup of $S$. If $e, f \in E(S)$ then, by (A1*), (A2), (A3) and duality,

$$
e^{*}=e^{*} e^{* *} e^{*}=e^{*} e^{*} e^{* *}=(e e)^{*} e^{* *}=e e^{* *}=f^{* *} f^{*}=f^{*}
$$

so $e \rho f$. Since $S$ is regular this proves $S^{*} \equiv S / \rho$ is a group. By the proof of Lemma 3.2, using (A1*) in place of (A1), (A7) holds, so for all $s, t \in S$, we have $s \rho s^{* *}$ and

$$
s \rho t^{*} \Rightarrow s^{*}=t^{* *} \Rightarrow t^{*}=t^{* *}=s^{* *},
$$

which proves $S^{*}$ is a transversal for $\rho$.

We have seen in the preceding section that the group transversal was actually a maximal subgroup of $S$ and the corresponding congruence was the least group congruence. This is generally not the case in the present context. First we may take a trivial subgroup getting the universal congruence for the group congruence. Conversely, in a completely simple nonorthodox semigroup $S$, the unit class of the least group congruence $\sigma$ on $S$ contains nonidempotents since $S$ is not $E$-unitary. If $a$ is such a nonidempotent and 
$a \in H_{e}$ where $e \in E(S)$, then $a \sigma e$ so $\left.\sigma\right|_{H_{e}}$ is not the equality relation on $H_{e}$. So if $G$ is a subgroup of $H_{e}$ and a transversal of $\sigma, G$ must be a proper subgroup of $H_{e}$.

Acknowledgement. We are indebted to the referee for careful reading of the paper.

\section{References}

[1] Blyth, T.S., Giraldes, E., Marques-Smith, M.P.O. (1994). Associate subgroups of orthodox semigroups. Glasgow Math. J. 36:163-171.

[2] Blyth, T.S., McFadden, R. (1983). On the construction of a class of regular semigroups. J. Algebra 81(1):1-22.

[3] Blyth, T.S., McFadden, R. (1983). Unit orthodox semigroups. Glasgow Math. J. 24:39-42.

[4] Blyth, T.S., Mendes Martins, P. (1997). On associate subgroups of regular semigroups. Comm. Algebra 25(7):2147-2156.

[5] Edwards, C.C. (1980). F-regular and F-orthodox semigroups. Semigroup Forum 19:331-345.

[6] Petrich, M., Reilly, N.R. (1999). Completely regular semigroups. New York: Wiley. 